\title{
Evaluación de la Actividad Antioxidante de Aceites Esenciales de Eucaliptos Cultivados en Colombia
}

\author{
Efrain A. Boom ${ }^{(1,2) *}$, José A. Orozco(1), Jader D. Alean ${ }^{(2)}$ y Benjamín Rojano(3) \\ (1) Universidad Popular del Cesar, Salida a Patillal, Valledupar-Colombia. \\ (2) Universidad de La Guajira, Km 5 salida Maicao, Riohacha-Colombia. \\ (3) Escuela de Química, Universidad Nacional de Colombia, Medellín- Colombia. \\ (e-mail: ingenieroboom@gmail.com; joseaoo@yahoo.es; jdalean@uniguajira.edu.co; brojano@unal.edu.co)
}

${ }^{*}$ Autor a quien debe ser dirigida la correspondencia.

Recibido Mar. 13, 2018; Aceptado May. 22, 2018; Versión final Jul. 20, 2018, Publicado Dic. 2018

\begin{abstract}
Resumen
En este trabajo se evaluó la actividad antioxidante de aceites esenciales obtenidos a partir de eucaliptos sembrados en el Departamento de Cesar (Colombia). Los rendimientos de extracción de los aceites esenciales oscilan entre $0.59-1.24 \%$. La composición química de los aceites esenciales de las hojas de eucalipto (tereticornis y camaldulensis) fue evaluada por cromatografía de gases acoplada a espectrometría de masas. Los principales componentes de los aceites esenciales fueron el eucaliptol (41-50\%), $\alpha$-pineno (7$13 \%)$, $\alpha$-terpineol (3-7\%) y $\beta$-pineno (2-4\%). La actividad antioxidante se determinó utilizando las técnicas de fenoles totales $(600-950 \mathrm{mg} / \mathrm{L})$, FRAP $(600-2500 \mathrm{mg} / \mathrm{L})$, DPPH $(440-600 \mu \mathrm{mol} / \mathrm{L})$ y ORAC $(60000-120000$ $\mu \mathrm{mol} / \mathrm{L})$.
\end{abstract}

Palabras clave: aceite esencial; eucalipto; capacidad antioxidante

\section{Evaluation of Antioxidant Activity of Eucalyptus Essential Oils Grown in Colombia}

\begin{abstract}
In this work, the antioxidant activity of essential oils obtained from eucalyptus seed in the Cesar Department (Colombia) was evaluated. The extraction yields of essential oils varied between $0.59-1.24 \%$. The chemical composition of essential oils from the eucalyptus leaves (tereticornis and camaldulensis) was evaluated by Gas Chromatography-Mass Spectrometry (GC-MS). The main components of the essential oils were eucalyptol (41-50\%), $\alpha$-pinene (7-13\%), $\alpha$-terpineol (3-7\%) and $\beta$-pinene (2-4\%). The antioxidant activity was determined by the techniques of Total Phenols $(600-950 \mathrm{mg} / \mathrm{L})$, FRAP $(600-2500 \mathrm{mg} / \mathrm{L})$, DPPH $(440-600$ $\mu \mathrm{mol} / \mathrm{L})$ and ORAC (60000-120000 $\mu \mathrm{mol} / \mathrm{L})$.
\end{abstract}

Keywords: essential oil; eucalyptus; antioxidant capacity 


\section{INTRODUCCION}

En los últimos años, la industria alimentaria y los consumidores han expresado el deseo de reducir el uso de productos sintéticos para la conservación de alimentos; una de las tendencias actuales es el uso de metabolitos secundarios (terpenos, compuestos fenólicos, glúcidos y alcaloides) y compuestos bioactivos de origen natural. El eucalipto es una planta que se puede aprovechar industrialmente debido a que las glándulas de las hojas segregan aceites esenciales (Moreno et al., 2010). En la industria alimentaria, los aceites esenciales se utilizan en los condimentos, en confitería actúan como refrescantes y en las drogas enmascaran sabores amargos. En la industria no alimentaria son de gran utilidad en los cigarros, perfumes, papeles y productos cosméticos; también tiene la capacidad de enmascarar olores en pinturas, textiles y plásticos.

La capacidad antioxidante que tiene una matriz biológica está representada por el contenido de metabolitos secundarios (Alean et al., 2016). Estudios han demostrado el potencial anticancerígeno de los extractos de eucalipto contra algunas células cancerosas del colon, páncreas, pulmón, próstata, ovario, cuello uterino, hígado y neuroblastoma (Al-Sayed et al., 2012; Islam et al., 2012; Vuong et al., 2015). Marzoug et al., (2011) determinó la capacidad antioxidante del aceite esencial de eucalipto oleosa con las técnicas de DPPH (2,2difenil-1-picrilhidrazilo) y ABTS (2,2'-azino-bis-83-ethylbenzothiazoline-6-sulphonic acid). Los resultados para DPPH fueron una consecuencia de la baja cantidad de metabolitos presentes en todas las muestras. Sin embargo, con el ensayo ABTS la parte más activa se presentó en las hojas con un valor $I_{50}$ de 13,0 mg/L, seguido de los tallos con $\mathrm{IC}_{50}$ de $43,5 \mathrm{mg} / \mathrm{L}$ (Marzoug et al., 2011). Los principales compuestos que contribuyen a la actividad antioxidante del aceite esencial de las hojas del eucalipto son los compuestos terpénicos (Lima et al., 2013; Yáñez y Cuadro, 2012); en la Tabla 1, se puede observar los compuestos reportados en la literatura con mayor presencia en las hojas de eucalipto.

Tabla 1: Compuestos presentes en algunas especies de eucalipto.

\begin{tabular}{|c|c|c|c|}
\hline Eucalipto & Principales compuestos & Lugar & Autores \\
\hline \multirow{6}{*}{ Camaldulensis } & $\begin{array}{l}\text { Eucaliptol (46.74\%), aromadendreno }(12.1 \%) \text {, terpinen-4-ol } \\
(7.6 \%) \text { y } \alpha \text {-pineno }(6.35 \%) .\end{array}$ & Irán & Lima et al., (2013) \\
\hline & $\begin{array}{l}\text { Eucaliptol }(29.2 \%) \text {, a-felandreno }(17.43 \%) \text {, a-pineno }(7.1 \%) \text {, } \\
\text { aromadendreno }(5.75 \%) \text { y terpinen-4-ol }(4.92 \%)\end{array}$ & Irán & Lima et al., (2013) \\
\hline & $\begin{array}{l}\text { Eucaliptol }(77.41 \%) \text {, terpinen-4-ol }(3.68 \%) \text {, a-pineno }(3.64 \%) \text {, } \\
\text { limoneno }(3.21 \%) \text { y } \beta \text {-mirceno }(1.41 \%) \text {. }\end{array}$ & Colombia & $\begin{array}{l}\text { Yáñez y Cuadro, } \\
\text { (2012) }\end{array}$ \\
\hline & $\begin{array}{l}\text { Terpinen-4-ol (10.24\%), eucaliptol }(7.89 \%) \text {, } \alpha \text {-terpineno } \\
(6.93 \%) \text {, a-felandreno }(4.01 \%) \text {, carvacrol }(3.87 \%) \text {, terpineol } \\
(3.3 \%) \text {, citronelol }(3.22 \%), \beta \text {-citronelol }(3.22 \%) \text {, timol }(2.19 \%) \text {, } \\
\beta \text {-cariofileno }(1.83 \%) \text { y citronelal }(0.79 \%) ;\end{array}$ & Guatemala & $\begin{array}{l}\text { De León Juárez, } \\
\text { (2008) }\end{array}$ \\
\hline & $\begin{array}{l}\text { Y-terpineno }(71.36 \%) \text {, eucaliptol }(0.46 \%) \text {, o-cimeno }(17.63 \%) \text {, } \\
\text { terpinoleno }(1.10 \%) \text { y } \alpha \text {-pineno }(0.54 \%) \text {. }\end{array}$ & Malasia & $\begin{array}{l}\text { Mubarak et al., } \\
(2014)\end{array}$ \\
\hline & $\begin{array}{l}\text { p-cimeno (17.38 a } 28.60 \%), \beta \text {-felandreno }(12.35-14.47 \%), \beta- \\
\text { pineno }(0.94-11.48 \%) \text {, criptona }(4.97-7.25 \%) \text {, terpinen-4-ol- } \\
4.21 \%) \text {, espatulenol }(7.83-14.15 \%) \text {, } \alpha \text {-pineno }(1.66-5.01 \%) \text { y } \\
\text { eucaliptol }(1.78-2.89 \%) \text {. }\end{array}$ & $\begin{array}{l}\text { República } \\
\text { de Serbia }\end{array}$ & $\begin{array}{l}\text { Grbović, Orčić, y } \\
\text { Couladis, (2010) }\end{array}$ \\
\hline \multirow[b]{2}{*}{ Tereticornis } & a-Pineno (30.1\%), eucaliptol (21.8\%). & India & Kaur et al., (2011) \\
\hline & $\begin{array}{l}\text { Citronelal ( } 44.8 \%) \text {, citronelol }(9.78 \%) \text {, ácido citronelico }(6.47 \%) \\
\text { y eucaliptol }(3.10 \%) \alpha \text {-pineno }(1.58 \%) \text {. }\end{array}$ & Colombia & Murillo et al., (2011) \\
\hline Citriodora & $\begin{array}{l}\text { Timol }(10.9 \%) \text {, citronelol }(39.27 \%) \text {, citronelal }(18.9 \%) \text { y } \beta- \\
\text { cariofileno }(2.62 \%) \text {. }\end{array}$ & Guatemala & $\begin{array}{l}\text { De León Juárez, } \\
\text { (2008) }\end{array}$ \\
\hline \multirow[t]{2}{*}{ Globulus } & $\begin{array}{l}\text { Eucaliptol (82.27\%), limoneno (3.7\%), a-pineno (3.16\%), guaiol } \\
(2.76 \%) \text {, terpinen-4-ol (1.4\%) y linalol (1.3\%). }\end{array}$ & Colombia & $\begin{array}{l}\text { Yáñez y Cuadro, } \\
\text { (2012) }\end{array}$ \\
\hline & Eucaliptol (75\%). & Uruguay & Mantero et al., 2007 \\
\hline
\end{tabular}

A parte de los metabolitos secundarios pertenecientes a la familia de los terpenos, la literatura reporta la presencia de otros metabolitos (compuestos pertenecientes a la familia de los compuestos fenólicos) tales como ácido gálico, ácido protocatequídico, ácido elágico, quercetina, glicósido de quercetina, naringenina, catequina, epicatequina, rutina, quercitrina, apigenina y miricetina (Al Sayed et al., 2012; Bhuyan et al., 2015; Vázquez et al., 2012; Santos et al., 2012). Este trabajo tiene como objetivo evaluar la capacidad antioxidante de los aceites esenciales del eucalipto cultivado en tres municipios del departamento de Cesar (Colombia). 


\section{MATERIALES Y MÉTODOS}

En este trabajo las especies de estudio fueron E. camaldulensis y E. tereticornis, sembradas en tres lugares diferentes en la zona de minería del departamento del Cesar. La primera proveniente de la zona rural del municipio de Chiriguaná con una altura de 106 msnm, la segunda del municipio de El Paso ubicado a 206 msnm y la tercera proveniente de la zona rural del municipio de La Jagua de Ibiríco con una altura de 151 msnm; todas cultivadas en clima seco. Las muestras objeto de estudio fueron hojas verdes de la parte baja de los arboles; cada muestreo se realizó por triplicado y por cuarteo al azar en las plantaciones. La recolección de las hojas frescas se realizó en árboles en pie, la edad de estos árboles en todos los lugares era de 6 años.

La cantidad de material vegetal para el proceso de extracción de aceites esenciales fue de $300 \mathrm{~g}$ (peso fresco) variando en proporción el volumen de agua para la extracción. Se realizó lavado y reducción del material vegetal por corte mecánico; la hidrodestilación se llevó a cabo en un equipo clevenger a nivel de laboratorio; se utilizó un intervalo de temperatura entre $96-112^{\circ} \mathrm{C}$, relaciones masa/volumen ( $\mathrm{g}$ de hojas de eucalipto/ml de $\mathrm{H}_{2} \mathrm{O}$ ) de 1:5, 1:6 y 1:8. Para retirar el exceso de agua generado, los extractos se centrifugaron y se les adicionó $\mathrm{Na}_{2} \mathrm{SO}_{4}$. La densidad obtenida para el aceite esencial de eucalipto camaldulensis fue $0.908 \mathrm{~g} / \mathrm{ml}$ y para el eucalipto tereticornis es $0.884 \mathrm{~g} / \mathrm{ml}$. Se realizaron análisis cromatográficos y espectrofotométricos; con el fin de determinar los compuestos que caracterizaban los extractos como aceites esenciales y se evaluó la capacidad antioxidante de los mismos.

Cromatografía de gas y cromatografía de gas-espectrometría de masas (GC y GC-MS)

Los aceites esenciales obtenidos se caracterizaron en un cromatógrafo de gases Agilent $6890 \mathrm{~N}$ y detector selectivo de masas Agilent 5973N (GC-MS). Se usó una columna capilar Agilent 123_5536DB_5MS (30 m $x 0.32 \mathrm{~mm}$ d.i, $0.5 \mu \mathrm{m}$ film). El programa de temperatura utilizado fue de $50^{\circ} \mathrm{C}-350^{\circ} \mathrm{C}$; tiempo inicial $1 \mathrm{~min}$ con equilibrio a $0.5 \mathrm{~min}$, temperatura del inyector de $175^{\circ} \mathrm{C}$, helio como gas portador, sistema de inyección Splitless y volumen de inyección 5.00 microlitros. La identificación y cuantificación de los compuestos característicos fue realizada según los espectros de masas, usando la base de datos que presentaba una mayor probabilidad de coincidencia. La identificación y cuantificación de los compuestos característicos fue realizada según los espectros de masas, usando la base de datos NIST98.L, NIST02.L y NIST5a.L, que presentaban una mayor probabilidad de coincidencia. Las condiciones de operación fueron de $350{ }^{\circ} \mathrm{C}$ de temperatura, con un modo de flujo constante, el flujo inicial fue de $1.2 \mathrm{ml} / \mathrm{min}$ y la presión inicial de 0.28 psi y una velocidad de $40 \mathrm{~cm} / \mathrm{s}$.

\section{Fenoles totales}

El contenido de fenoles totales se determinó de acuerdo con el método adaptado de Folin-Ciocalteu (Singleton y Rossi, 1965). Los extractos $(50 \mathrm{ml})$ se mezclaron con $125 \mathrm{ml}$ de reactivo de Folin-Ciocalteu y $400 \mathrm{ml}$ de solución de carbonato sódico $(7.1 \% \mathrm{p}=\mathrm{v})$ y la solución resultante se llevó a un volumen final de $1000 \mathrm{ml}$. La mezcla se agitó y se almacenó a temperatura ambiente durante 30 minutos a oscuras. La absorbancia se midió a $760 \mathrm{~nm}$ frente a un blanco. Se usaron soluciones acuosas de ácido gálico para la calibración, los resultados se expresan como mg equivalentes de ácido gálico.

\section{Poder antioxidante reductor del hierro (FRAP)}

La capacidad antioxidante de los aceites esenciales de eucalipto se estimó según el procedimiento descrito por Benzie y Straind (1996). Este método se basa en el aumento de la absorbancia debido a la formación de 2,4,6-tripiridil-s-triazina (TPTZ) - Fe (II) en presencia de agentes reductores. Se utilizaron $900 \mu \mathrm{L}$ de una solución de TPTZ, $50 \mu \mathrm{L}$ de muestra y $50 \mu \mathrm{L}$ de agua destilada. Luego de $30 \mathrm{~min}$ se leyó la absorbancia a una longitud de onda de $593 \mathrm{~nm}$. Los valores de FRAP se expresaron como AEAC (capacidad antioxidante equivalente de ácido ascórbico: $\mathrm{mg}$ de ácido ascórbico por $100 \mathrm{~g}$ de polvo seco) usando una curva estándar de ácido ascórbico (Zapata et al., 2015).

\section{Método DPPH (2,2-difenil-1-picrilhidrazilo)}

El 1,1-difenil-1-picrilhidrazilo ( $\left.\mathrm{DPPH}{ }^{\bullet}\right)$, es un radical libre estable que presenta una coloración púrpura en medio metanólico, el cual se midió usando los métodos de Brand-Williams (Brand-Williams et al., 1995), con algunas modificaciones. El método se basa en la reacción de $10 \mathrm{ml}$ de muestra con $990 \mathrm{ml}$ de solución de DPPH por 30 min a temperatura ambiente, seguido de la determinación de la disminución de la absorbancia a $517 \mathrm{~nm}$ asociada con una reducción de la concentración de DPPH. Los resultados se expresaron en unidades Trolox equivalentes antioxidante (TEAC) (Rojano et al., 2008). 


\section{Capacidad de absorción de radicales de oxígeno (ORAC)}

El ensayo de ORAC se determinó usando la siguiente metodología: Se prepararon $3 \mathrm{ml}$ a partir de la siguiente solución: $21 \mu \mathrm{l}$ de una solución de fluoresceína $10 \mu \mathrm{M}, 2899 \mu \mathrm{L}$ de buffer fosfato $75 \mathrm{mM}(\mathrm{pH}$ 7.4), $50 \mu \mathrm{l}$ de AAPH 600 mM (2,20-Azo-bis (2-amidinopropano) dihidrocloruro) y $30 \mu \mathrm{L}$ de muestra o Trolox $500 \mu \mathrm{M}$ (estándar). La fluorescencia se registró en un espectrofluorómetro Perkin Elmer LS55 con una multicelda termostatizada. EI valor ORAC $\mu$ molTrolox/100 g liofilizado se calculó mediante una curva de calibración utilizando diferentes concentraciones de Trolox ${ }^{\circledR}$ (Zapata et al., 2013).

\section{RESULTADOS Y DISCUSION}

Para la relación masa-volumen ( $\mathrm{g}$ de hojas de eucalipto/ml de $\mathrm{H}_{2} \mathrm{O}$ ) de $1: 5$ se realizó un análisis de varianza (ANOVA) en statgraphics ${ }^{\circledR}$. El análisis ANOVA confirma que no existen diferencias estadísticas significativas con un valor $\mathrm{P} \leq 0.05$, entre las muestras estudiadas. Por tal razón, se realizó un análisis de múltiples rangos Fisher's Least Significant Difference (LSD). El resultado de este análisis reveló que las muestras se pueden agrupar en un grupo homogéneo con características similares (tabla 2). Los rendimientos de extracción que conforman el mismo grupo homogéneo no tienen diferencias estadísticamente significativas; por lo tanto, los rendimientos de los aceites esenciales de El Paso, Chiriguaná y La Jagua de Ibiríco, son equivalentes con un $95 \%$ de confianza (tabla 2). En la figura 1 se ilustra lo expresado en la tabla 2; en ese caso se observa que las barras de error de los rendimientos de extracción de los diferentes municipios se solapan, lo que significa que los valores no son estadísticamente diferentes cuando se maneja una relación de masa volumen de 1:5.

Tabla 2: Medias y múltiples rangos por especie correspondiente al diseño masa/volumen (1:5)

\begin{tabular}{|l|c|c|c|c|c|}
\hline \multicolumn{1}{|c|}{ Nivel } & Casos & Media & Grupos Homogéneos & Límite Inferior & Límite Superior \\
\hline E. tereticornis - El Paso & 3 & 0.5993 & $\mathrm{X}$ & 0.496524 & 0.702142 \\
\hline E. camaldulensis - Chiriguaná & 3 & 0.7773 & $\mathrm{X}$ & 0.674524 & 0.880142 \\
\hline E. camaldulensis - La Jagua de Ibiríco & 3 & 0.6560 & $\mathrm{X}$ & 0.553191 & 0.758809 \\
\hline
\end{tabular}

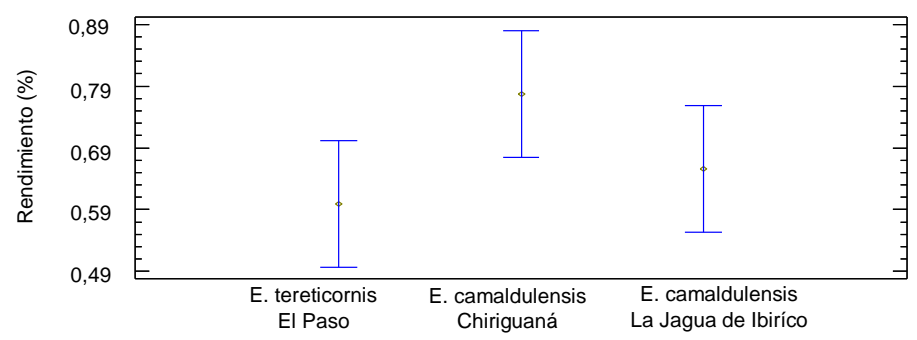

Fig. 1: Diagrama de cajas para la extracción de aceite esencial correspondiente al diseño masa/volumen (1:5)

En el análisis de varianza para la relación masa-volumen de 1:6, se identificó que existe diferencia significativa entre las muestras con un 95\% de confianza. El resultado reveló que las muestras se pueden agrupar en dos grupos homogéneo con características similares: grupo uno, aceite esencial del El Paso y La Jagua de lbiríco y grupo dos, aceite esencial de Chiriguaná (tabla 3). En la figura 2 se ilustra lo expresado en la tabla 3; en ese caso se observa que las barras de error de los rendimientos de extracción de los diferentes municipios solo se solapan entre las muestras de EI Paso y La Jagua de Ibiríco, lo que significa que los valores entre estos dos no son estadísticamente diferentes cuando se maneja una relación de masa volumen de 1:6.

Tabla 3: Medias y múltiples rangos por especie correspondiente al diseño masa/volumen (1:6)

\begin{tabular}{|l|c|c|c|c|c|}
\hline \multicolumn{1}{|c|}{ Nivel } & Casos & Media & Grupos Homogéneos & Límite Inferior & Límite Superior \\
\hline E. tereticornis - El Paso & 3 & 0.953667 & $\mathrm{X}$ & 0.866996 & 1.04034 \\
\hline E. camaldulensis - Chiriguaná & 3 & 1.241670 & $\mathrm{X}$ & 1.155000 & 1.32834 \\
\hline E. camaldulensis - La Jagua de Ibiríco & 3 & 0.918667 & $\mathrm{X}$ & 0.831996 & 1.00534 \\
\hline
\end{tabular}

En el análisis de varianza para la relación masa-volumen de 1:8, se identificó que existe diferencia significativa entre las muestras con un $95 \%$ de confianza. El resultado reveló que las muestras se pueden agrupar en dos grupos homogéneos según la alineación de las X's en columnas y que no existen diferencias estadísticamente significativas entre aquellos niveles que comparten una misma columna de X's (tabla 4). En la figura 3 se ilustra lo expresado en la Tabla 4; en ese caso se observa que las barras de error del rendimiento de extracción de La Jagua de Ibiríco se solapan con los otros dos rendimientos, lo que significa que los otros valores no son estadísticamente diferentes cuando se comparan con el de La Jagua de Ibiríco. Este comportamiento estadístico se puede presentar cuando los límites superiores e inferiores son muy grandes; en este caso se observa que son superiores a 1 (tabla 4). 


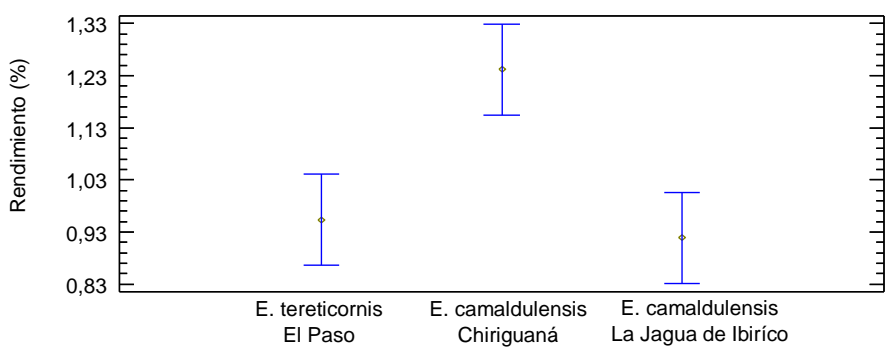

Fig. 2: Gráfico de las medias en el rendimiento correspondiente al diseño masa/volumen (1:6)

Tabla 4: Medias y múltiples rangos por especie correspondiente al diseño masa/volumen (1:8)

\begin{tabular}{|l|c|c|c|c|c|}
\hline Nivel & Casos & Media & Grupos Homogéneos & Límite Inferior & Límite Superior \\
\hline E. tereticornis - El Paso & 3 & 1.08133 & $\mathrm{X}$ & 1.02550 & 1.13716 \\
\hline E. camaldulensis -Chiriguaná & 3 & 1.21133 & $\mathrm{X}$ & 1.15550 & 1.26716 \\
\hline E. camaldulensis - La Jagua de Ibiríco & 3 & 1.15067 & $\mathrm{XX}$ & 1.09484 & 1.20650 \\
\hline
\end{tabular}

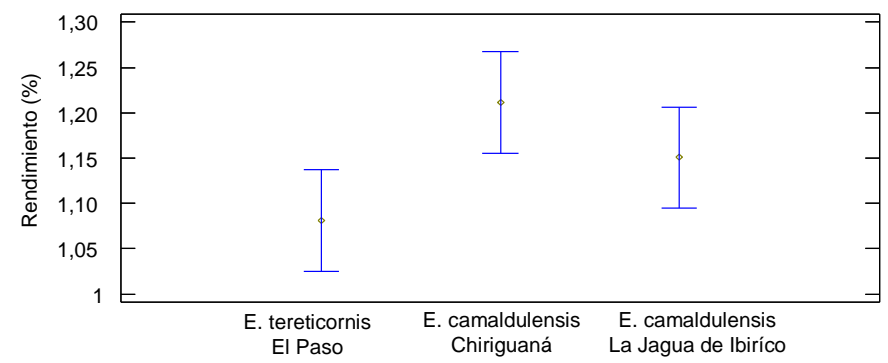

Fig. 3: Gráfico de las medias en el rendimiento correspondiente al diseño masa/volumen (1:8)

En la Tabla 5, 6 y 7 se presentan los principales compuestos de mayor importancia identificados en cada una de las variedades de eucaliptos evaluadas, con los tiempos de retención de cada compuesto y la base de datos y bibliotecas que permitieron la identificación de los diferentes componentes de los aceites esenciales. Para el E. camaldulensis de Chiriguaná, los compuestos de mayor concentración fueron eucaliptol, $\alpha$-pineno, isocarveol y $\alpha$-terpineol. Para E. camaldulensis de La Jagua de lbiríco los compuestos de mayor concentración fueron eucaliptol, $\alpha$-pineno, $\alpha$-terpineol, $\gamma$-terpineno y azuleno.

Para el E. tereticornis de EI Paso, los compuestos de mayor concentración fueron eucaliptol, $\alpha$-pineno, $\alpha$ terpineol, $y$-terpineno y ledol. Los tiempos de retención de los compuestos identificados; se encontraron dentro de los reportados por la literatura para el $\alpha$-pineno (5-20 $\mathrm{min}), \beta$-pineno $(6-15 \mathrm{~min})$, eucaliptol (6-18 $\mathrm{min}), \alpha-$ terpineol (9-35 $\mathrm{min}), \mathrm{v}$-terpineno $(16 \mathrm{~min}$ ) y globulol (35 $\mathrm{min}$ ) en aceites esenciales de especies de eucalipto como tereticornis, citriodora, camaldulensis y globulus (Guleria et al., 2012; Singh et al., 2012; Granados et al., 2014; Guala et al., 2014; Granados et al., 2015; Zambón et al., 2015; González-Guiñez et al., 2016).

Tabla 5: Principales compuestos, tiempo de retención y porcentaje relativo en E. camaldulensis de Chiriguaná

\begin{tabular}{|c|c|c|c|c|c|}
\hline $\begin{array}{l}\text { Tiempo de } \\
\text { retención (min) }\end{array}$ & $\begin{array}{l}\text { Compuesto } \\
\text { químico }\end{array}$ & $\begin{array}{l}\text { Porcentaje } \\
\text { relativo (\%) }\end{array}$ & $\begin{array}{l}\text { Porcentaje } \\
\text { relativo (\%) }\end{array}$ & $\begin{array}{l}\text { Probabilidad coincidencia } \\
\text { con la base de datos (\%) }\end{array}$ & Base de datos \\
\hline 9.462 & \multirow{3}{*}{ a-pineno } & 3.41 & \multirow{3}{*}{11.56} & \multirow{3}{*}{96} & NIST02.L \\
\hline 9.814 & & 1.30 & & & NIST02.L \\
\hline 10.853 & & 6.85 & & & NIST02.L \\
\hline 12.668 & $\beta$-pineno & 2.49 & 2.49 & 94 & NIST02.L \\
\hline 15.372 & \multirow{2}{*}{ Eucaliptol } & 34.50 & \multirow{2}{*}{49.88} & \multirow{2}{*}{96} & NIST02.L \\
\hline 16.007 & & 15.33 & & & NIST02.L \\
\hline 23.433 & Isocarveol & 3.55 & 3.55 & 83 & NIST02.L \\
\hline 23.829 & a-terpineol & 3.14 & 3.14 & 91 & NIST02.L \\
\hline 42.504 & Epiglobulol & 1.26 & 1.26 & 99 & NIST02.L \\
\hline 43.499 & (-) -Globulol & 2.73 & 2.73 & 96 & NIST02.L \\
\hline
\end{tabular}


Tabla 6: Principales compuestos, tiempo de retención y porcentaje relativo en E. camaldulensis de La Jagua de Ibiríco

\begin{tabular}{|c|c|c|c|c|c|}
\hline $\begin{array}{l}\text { Tiempo de } \\
\text { retención (min) }\end{array}$ & $\begin{array}{l}\text { Compuesto } \\
\text { químico }\end{array}$ & $\begin{array}{l}\text { Porcentaje } \\
\text { relativo (\%) }\end{array}$ & $\begin{array}{l}\text { Porcentaje } \\
\text { relativo (\%) }\end{array}$ & $\begin{array}{l}\text { Probabilidad coincidencia } \\
\text { con la base de datos (\%) }\end{array}$ & Base de datos \\
\hline 9.461 & \multirow{2}{*}{ a-pineno } & 3.76 & \multirow{2}{*}{13.0} & \multirow{2}{*}{97} & NIST5a.L \\
\hline 10.871 & & 9.24 & & & NIST5a.L \\
\hline 11.505 & $\beta$-pineno & 2.65 & 2.65 & 91 & NIST5a.L \\
\hline 15.355 & Eucaliptol & 41.83 & 41.83 & 96 & NIST5a.L \\
\hline 16.694 & $\mathrm{y}$-terpineno & 2.70 & 2.70 & 94 & NIST5a.L \\
\hline 19.636 & Fenchol & 2.02 & 2.02 & 98 & NIST5a.L \\
\hline 23.882 & a-terpineol & 6.79 & 6.79 & 90 & NIST5a.L \\
\hline 43.499 & Azuleno & 2.22 & 2.22 & 96 & NIST5a.L \\
\hline
\end{tabular}

Tabla 7: Principales compuestos, tiempo de retención y porcentaje relativo en E. tereticornis de El Paso

\begin{tabular}{|c|c|c|c|c|c|}
\hline $\begin{array}{l}\text { Tiempo de } \\
\text { retención (min) }\end{array}$ & $\begin{array}{l}\text { Compuesto } \\
\text { químico }\end{array}$ & $\begin{array}{l}\text { Porcentaje } \\
\text { relativo (\%) }\end{array}$ & $\begin{array}{l}\text { Porcentaje } \\
\text { relativo (\%) }\end{array}$ & $\begin{array}{l}\text { Probabilidad coincidencia } \\
\text { con la base de datos (\%) }\end{array}$ & Base de datos \\
\hline 9.426 & \multirow{2}{*}{ a-pineno } & 2.54 & \multirow{2}{*}{7.46} & \multirow{2}{*}{97} & NIST02.L \\
\hline 10.844 & & 4.92 & & & NIST02.L \\
\hline 12.677 & $\beta$-pineno & 3.87 & 3.87 & 94 & NIST02.L \\
\hline 15.337 & Eucaliptol & 49.17 & 49.17 & 98 & NIST02.L \\
\hline 16.667 & $\mathrm{y}$-terpineno & 3.20 & 3.20 & 94 & NIST02.L \\
\hline 22.481 & Isoborneol & 1.57 & 1.57 & 87 & NIST5a.L \\
\hline 23.873 & a-terpineol & 4.62 & 4.62 & 90 & NIST98.L \\
\hline 43.508 & Ledol & 3.41 & 3.41 & 95 & NIST5a.L \\
\hline
\end{tabular}

La concentración de los compuestos identificados se comparó con otros trabajos y se encontró que algunos de ellos están por encima de los reportados en la literatura (Tabla 8).

Tabla 8: Comparación de los principales compuestos de los aceites esenciales obtenidos con otros trabajos

\begin{tabular}{|c|c|c|c|c|c|c|}
\hline \multirow{2}{*}{ Especies } & \multirow{2}{*}{ País } & \multirow{2}{*}{$\begin{array}{c}\text { Rendimiento } \\
(\%)\end{array}$} & \multicolumn{3}{|c|}{ Porcentaje relativo (\%) } & \multirow{2}{*}{ Autores } \\
\hline & & & $\alpha$-pineno & Eucaliptol & $\alpha$-terpineol & \\
\hline E. camaldulensis & Colombia & 1.17 & 11.56 & 49.83 & 3.14 & Fuente propia \\
\hline E. camaldulensis & Colombia & 0.74 & 15.65 & 41.83 & 6.79 & Fuente propia \\
\hline E. tereticornis & Colombia & 0.77 & 7.46 & 49.17 & 4.62 & Fuente propia \\
\hline E. camaldulensis & Malaysia & 1.40 & 21.53 & 61.51 & 0.10 & Mubarak et al., (2014) \\
\hline E. camaldulensis & Irán & -- & 6.35 & 46.74 & 0.88 & Lima et al., (2013) \\
\hline E. camaldulensis & Irán & -- & 7.10 & 29.20 & 2.06 & Lima et al., (2013) \\
\hline E. globulus & Colombia & 4.00 & 3.16 & 82.27 & 1.95 & Yáñez y Cuadro, (2012) \\
\hline E. camaldulensis & Colombia & 3.00 & 3.64 & 77.41 & 1.20 & Yáñez y Cuadro, (2012) \\
\hline E. tereticornis & Colombia & 0.89 & 1.58 & 3.10 & -- & Murillo et al., (2011) \\
\hline E. tereticornis & India & 1.21 & 30.1 & 21.80 & 2.36 & Kaur et al., (2011) \\
\hline E. camaldulensis & Turquía & -- & -- & 13.73 & -- & Akin et al., (2010) \\
\hline E. camaldulensis & Montenegro & 0.67 & 1.66 & 2.89 & 0.58 & Grbović et al., (2010) \\
\hline E. camaldulensis & Montenegro & 0.70 & 5.01 & 2.03 & 0.66 & Grbović et al., (2010) \\
\hline E. camaldulensis & Montenegro & 1.59 & 3.88 & 1.70 & 0.48 & Grbović et al., (2010) \\
\hline E. camaldulensis & Montenegro & 0.68 & 3.59 & 1.78 & 0.71 & Grbović et al., (2010) \\
\hline E. camaldulensis & Guatemala & 1.15 & -- & 7.89 & 3.33 & De León Juárez, (2008) \\
\hline E. globulus & Uruguay & 0.88 & 3.32 & 87.33 & -- & Mantero et al., (2007) \\
\hline E. globulus & Uruguay & 1.05 & 7.64 & 76.24 & -- & Mantero et al., (2007) \\
\hline E. globulus & Uruguay & 1.4 & 5.86 & 78.3 & -- & Mantero et al., (2007) \\
\hline E. grandis & Uruguay & 0.27 & 7.64 & 76.24 & -- & Mantero et al., (2007) \\
\hline
\end{tabular}




\section{Análisis estadístico de la capacidad antioxidante}

La capacidad antioxidante de los aceites esenciales está determinada por el contenido de metabolitos y el modo de acción de cada uno de ellos; para evaluar esto, se utilizaron los métodos de Fenoles Totales, FRAP, DPPH y ORAC. Para comparar los resultados obtenidos por cada método, se realizó un análisis de ANOVA en statgraphics ${ }^{\circledR}$ y prueba de rango múltiple para determinar si los valores medios eran significativamente diferentes; el método utilizado para esto fue la diferencia menos significativa (LSD) de Fisher. A pesar que los compuestos identificados con mayor presencia pertenecen a la familia de los terpenos (tabla 5,6 y 7 ), la literatura reporta en aceites esenciales del eucalipto la existencia de metabolitos asociados a la familia de compuestos fenólicos (Al-Sayed et al., 2012; Vázquez et al., 2012; Bhuyan et al., 2015). Por tal razón, la valoración de fenoles totales fue realizada a cada una de los aceites obtenidos, el mayor contenido se obtuvo para la muestra de E. camaldulensis de Chiriguaná, seguido por E. camaldulensis de La Jagua de lbiríco (figura 4). Ninguno de los niveles o intervalos de confianza se solapan, lo que significa que todos los resultados son estadísticamente diferentes con un $95 \%$ confianza (figura 5).

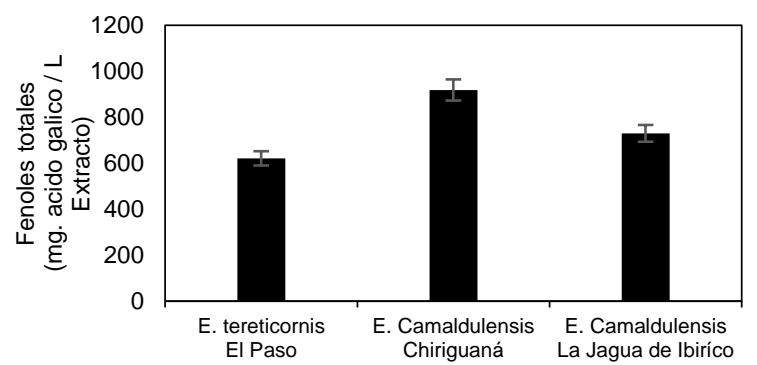

Fig. 4: Fenoles totales aceites esenciales de eucalipto

Los fenoles totales estuvieron por encima de los reportados por la literatura para otras especies de eucalipto, teniendo en cuenta que las densidades de los aceites esenciales de las especies estudiadas se encuentran entre 0.88-0.91 $\mathrm{g} / \mathrm{ml}$. Dezsi et al., (2015) determinaron fenoles totales para el aceite esencial de E. globulus de $235.87 \pm 4.38 \mathrm{mg} / \mathrm{g}$; Rahimi-Nasrabadi et al., (2013) para el aceite esencial de E. largiflorens encontraron fenoles totales constituyentes en la subfracción polar de $142.6 \pm 0.9 \mu \mathrm{g} / \mathrm{mg}$, subfracción no polar $68.6 \pm 0,4 \mathrm{mg} / \mathrm{g}$ y en el aceite esencial de $36,0 \pm 0,6 \mathrm{mg} / \mathrm{g}$.

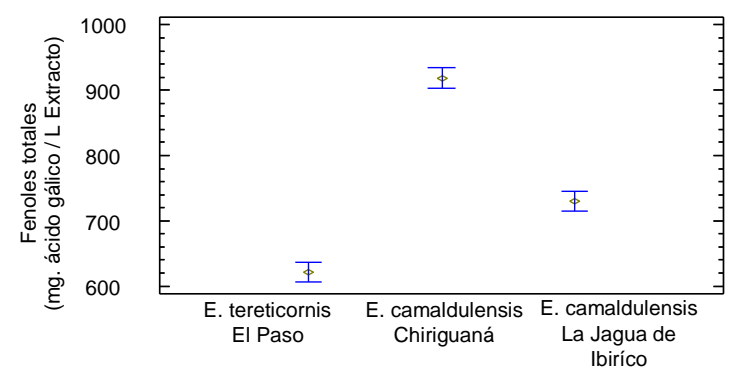

Fig. 5: Análisis de medias para fenoles totales por el método LSD.

En la figura 6 se observa que la mayor capacidad antioxidante al reducir un complejo férrico a su forma ferrosa la presenta la muestra de E. camaldulensis de Chiriguaná. Ninguno de los niveles o intervalos de confianza se solapan, lo que quiere decir que todos los resultados son estadísticamente diferentes con un $95 \%$ de confianza (figura 7). Los resultados de FRAP estuvieron por encima de los reportados por la literatura para otras especies de eucalipto (FRAP de $672.9 \mathrm{mg} / \mathrm{L}$ en aceite esencial de E. citriodora) (Singh et al., 2012).

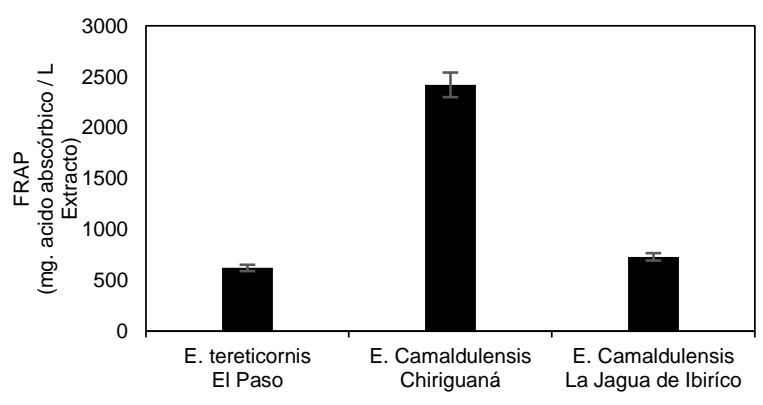

Fig. 6: FRAP aceites esenciales de eucalipto 


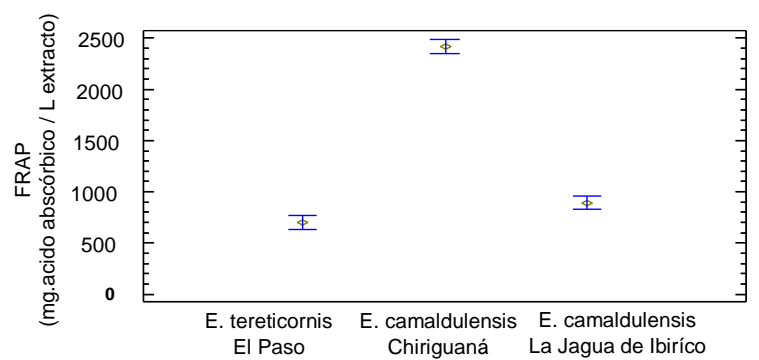

Fig. 7: Análisis de medias para FRAP por el método LSD

En la figura 8 se observa que la mayor capacidad antioxidante cuando existe una donación de un electrón o un protón $(\mathrm{DPPH} \bullet$ ) la presenta la muestra de E. camaldulensis de Chiriguaná. Se identifica que los niveles o intervalos de confianza del E. tereticornis de El Paso y el E. camaldulensis de La Jagua de lbiríco se solapan, lo que quiere decir que entre estos dos resultados no existe diferencias estadísticamente significativas con un 95\% de confianza (figura 9). Los resultados de DPPH estuvieron dentro del rango de los resultados reportados por la literatura para especies como E. camaldulensis (DPPH entre 500 y $5800 \mu \mathrm{mol} / \mathrm{L}$ ) (Barra et al., 2010).

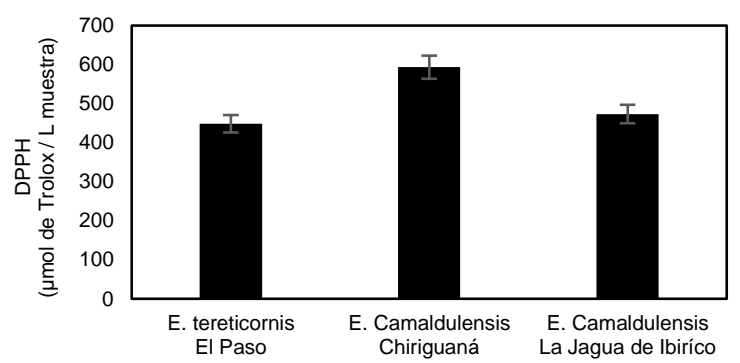

Fig. 8: DPPH aceites esenciales de eucalipto

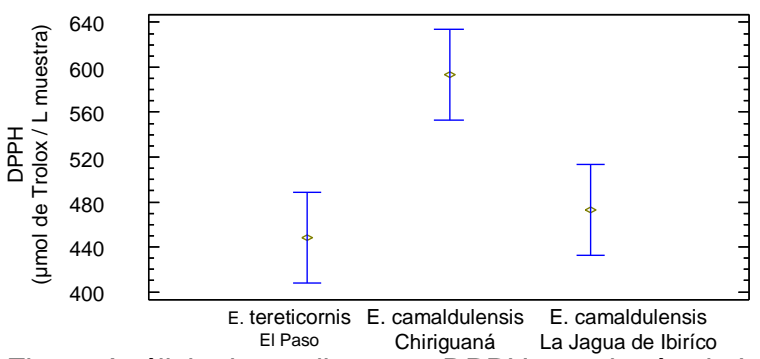

Fig. 9: Análisis de medias para DPPH por el método LSD

En la figura 10 se observa que la mayor capacidad antioxidante frente a radicales peróxilos (ORAC) la presenta la muestra de E. camaldulensis de La Jagua de Ibiríco. Los niveles o intervalos de confianza del E. tereticornis del Paso y el E. camaldulensis de Chiriguaná se solapan, lo que quiere decir que entre estos dos resultados no existen diferencias estadísticamente significativas con un 95\% de confianza (figura 11).

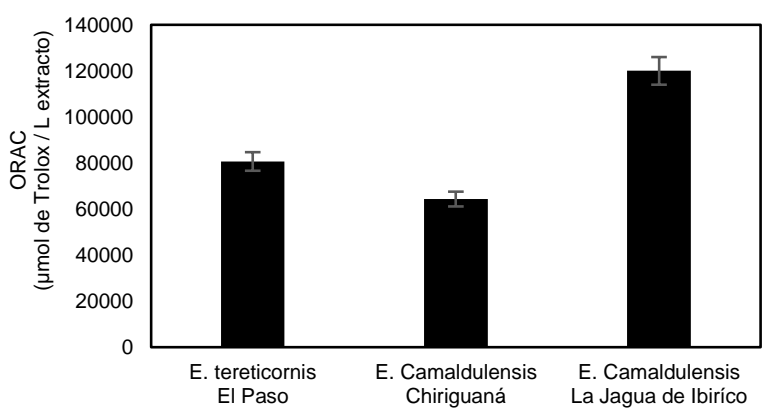

Fig. 10: ORAC aceites esenciales de eucalipto

A diferencia de los resultados registrados de la actividad antioxidante con los métodos de FRAP y DPPH, se observa que el E. camaldulensis de La Jagua de Ibiríco presenta una mejor actividad evaluada con el ORAC. Amakura et al., (2009) y Dudonne et al., (2009) reportaron valores de ORAC por encima de $4000 \mu \mathrm{molTE} / \mathrm{g}$ y $2846 \mu \mathrm{mol}$ Trolox/g para extractos de las hojas de E. globulus respectivamente. 


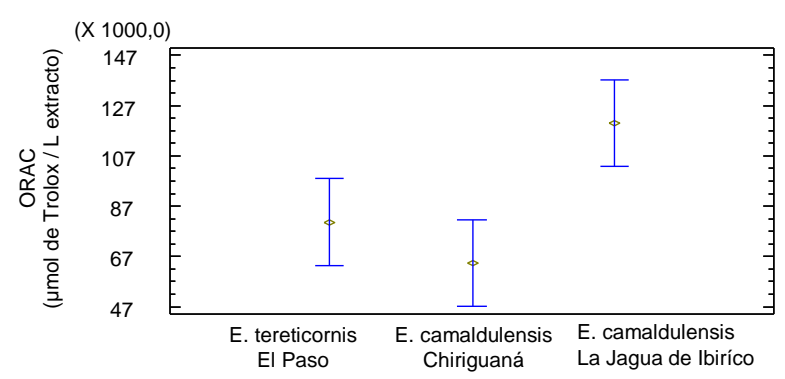

Fig. 11: Análisis de medias para ORAC por el método LSD

\section{CONCLUSIONES}

Los rendimientos de extracción de aceite esencial de las especies de eucalipto estudiadas fueron similares en las diferentes relaciones masa/volumen ( $\mathrm{g}$ de hojas de eucalipto/ $\mathrm{ml} \mathrm{de} \mathrm{H}_{2} \mathrm{O}$ ) establecidas. Los compuestos químicos de mayor concentración en las especies de eucalipto fueron el eucaliptol y el a-pineno, con valores superiores al $41 \%$ y al $7 \%$ respectivamente. Por otro lado, aparte de la identificación de terpenos se logró valorar la presencia de fenoles totales a partir de un monofenol (ácido gálico) como estándar de referencia. El aceite esencial extraído de las hojas de eucalipto camaldulensis procedente de Chiriguana, fue el que mejor actividad antioxidante presentó; seguido del aceite esencial procedente de La Jagua de lbiríco.

\section{REFERENCIAS}

Alean, J., F. Chejne y B. Rojano, Degradation of polyphenols during the cocoa drying process, doi: 10.1016/j.jfoodeng.2016.05.026, Journal of Food Engineering, 189, 99-105 (2016)

Akin, M., A. Aktumsek y A. Nostro, Antibacterial activity and composition of the essential oils of Eucalyptus camaldulensis Dehn. and Myrtus communis L. growing in Northern Cyprus, doi: 10.5897/AJB09.1562, 9(4), African Journal of Biotechnology, 531-535 (2010)

Al-Sayed, E., A. Singab y otros cuatro autores, HPLC-PDA-ESI-MS/MS profiling and chemopreventive potential of Eucalyptus gomphocephala DC, doi: 10.1016/j.foodchem.2011.09.036, Food Chemistry, 133, 1017-1024 (2012)

Amakura, Y., M. Yoshimura y otros tres autores, Marker Constituents of the Natural Antioxidant Eucalyptus Leaf Extract for the Evaluation of Food Additives, doi:10.1271/bbb.80832, Bioscience, Biotechnology, and Biochemistry, 73(5), 10601065 (2009)

Barra, A., V. Coroneo y otros tres autores, Chemical variability, antifungal and antioxidant activity of Eucalyptus camaldulensis essential oil from Sardinia, Natural Product Communications, 5(2), 329-335 (2010)

Benzie, I. y J. Straind, The ferric reducing ability of plasma (FRAP) as a measure of "antioxidant power": the FRAP assay, doi: 10.1006/abio.1996.0292, Analytical Biochemistry, 239(1), 70-76 (1996)

Bhuyan, D.J., Q. Van Vuong y otros cuatro autores, Microwave-assisted extraction of Eucalyptus robusta leaf for the optimal yield of total phenolic compounds, doi: 10.1016/j.indcrop.2015.02.044, Industrial Crops and Products, 69, 1-10 (2015)

Brand-Williams, W., M. Cuvelier y C. Berset, Use of a free radical method to evaluate antioxidant activity, doi: 10.1016/S0023-6438(95)80008-5, LWT - Food Science and Technology, 28, 25-30 (1995)

De León Juárez, M.L., Comparación del rendimiento de aceite esencial de dos especies de eucalipto (Eucalyptus citriodora hook y Eucalyptus camaldulensis Dehnh), aplicando el método de hidrodestilación a nivel laboratorio. Guatemala, Universidad de San Carlos de Guatemala, Facultad de Ingeniería, Escuela de Ingeniería Química (2008)

Dezsi, Ş., A. Bədərəu y otros cinco autores, L. Antimicrobial and antioxidant activities and phenolic profile of Eucalyptus globulus Labill. and Corymbia ficifolia (F. Muell.) K.D. Hill \& L.A.S. Johnson leaves, doi: 10.3390/molecules20034720, Molecules, 20(3), 4720-4734 (2015)

Dudonne, S., X. Vitrac y otros tres autores, Comparative Study of Antioxidant Properties and Total Phenolic Content of 30 Plant Extracts of Industrial Interest Using DPPH, ABTS, FRAP, doi: 10.1021/jf803011r, Comparative and General Pharmacology, C, 1768-1774 (2009)

González-Guiñez, R., G. Silva-Aguayo, A. Urbina-Parra y M. Gerding-González, Aceite esencial de Eucalyptus globulus Labill Y Eucalyptus nitens H. Deane \&amp; Maiden (myrtaceae) para el control de Sitophilus zeamais Motschulsky, Chilean Journal of Agricultural \& Animal Science | Ex Agro-ciencia, 32(3), 204-110 (2016)

Granados, C., G. Santafé y D. Acevedo, Chemical composition and evaluation of antioxidant activity of leaf essential oil Eucalyptus camaldulensis from Norte de Santander (Colombia), Revista U.D.C.A Actualidad \& Divulgación Científica, 18(1), 235-240 (2015)

Granados, C., X. Yáñez y D. Acevedo, Evaluación de la actividad antioxidante del aceite esencial foliar de myrcianthes leucoxyla de Norte de Santander (Colombia), doi: 10.4067/S0718-07642014000300003, Información Tecnológica, 25(3), $11-16(2014)$ 
Grbović, S., D. Orčić y M. Couladis, Variation of essential oil composition of Eucalyptus Camaldulensis (myrtaceae) from the Montenegro coastline, doi: 10.2298/APT1041151G, Acta periodica technologica, 41, 151-158 (2010)

Guala, M., M. Lapissonde, H. Elder y G.A. Pérez, Efecto acaricida del aceite esencial de aguaribay (schinus molle I.) y sus fracciones en colmenares de abejas (apis mellifera) en relación con la composición química, doi: 10.4067/S071807642014000200017, Información Tecnológica, 25(2), 151-156 (2014)

Guleria, S., A. Tiku y otros cuatro autores, Chemical composition, antioxidant activity and inhibitory effects of essential oil of Eucalyptus teretecornis grown in north-western Himalaya against Alternaria alternate, doi: 10.1007/s13562-011-00732, Journal of Plant Biochemistry and Biotechnology, 21(1), 44-50 (2012)

Islam, F., H. Khatun y otros tres autores, Bioassay of Eucalyptus extracts for anticancer activity against Ehrlich ascites carcinoma (eac) cells in Swiss albino mice, doi: 10.1016/S2221-1691(12)60063-X, Asian Pacific Journal of Tropical Biomedicine, 2, 394-398 (2012)

Kaur, S., H. Singh, D. Batish y R. Kohli, Chemical characterization, antioxidant and antifungal activity of essential oil from Eucalyptus tereticornis, Journal of Medicinal Plants Research, 5(19), 4786-4793 (2011)

Lima, L., B. Babakhani y otros tres autores, Essential oils composition and antibacterial activities of Eucalyptus camaldulensis Dehn., International Journal of Medicinal and Aromatic Plants, 3(2), 214-219 (2013)

Mantero, C., C. García y otros cuatro autores, Potencialidad para producción de aceites esenciales en especies de Eucalyptus cultivadas en Uruguay, Agrociencia, XI(2), 17 - 23 (2007)

Marzoug, H., M. Romdhane y otros seis autores, Eucalyptus oleosa essential oils: Chemical composition and antimicrobial and antioxidant activities of the oils from different plant parts (stems, leaves, flowers and fruits), doi:10.3390/MOLECULES16021695, Molecules, 16, 1695-1709 (2011)

Moreno, J., G. López y R. Siche, Modelación y optimización del proceso de extracción de aceite esencial de eucalipto (Eucalyptus globulus), doi: 10.17268/sci.agropecu.2010.02.05, Scientia Agropecuaria, 1, 147 - 154 (2010)

Mubarak, E., S. Mohajer, I. Ahmed y R. Taha, Essential Oil Compositions from Leaves of Eucalyptus camaldulensis Dehn. and Callistemon viminalis Originated from Malaysia, doi: 10.7763/IPCBEE. 2014. V70. 27, International Proceedings of Chemical, Biological \& Environmental, 70, 137-141 (2014)

Murillo, W., J. Acevedo Ruíz y C. Peláez, Fungicidal activity of Eucalyptus tereticornis essential oil on the pathogenic fungus Fusarium oxysporum, Revista Cubana de Farmacia, 45(2), 264-274 (2011)

Rahimi-Nasrabadi, M., S. Nazarian y otros cuatro autores, Chemical composition, antioxidant, and antibacterial activities of the essential oil and methanol extracts of Eucalyptus largiflorens F. Muell, doi: 10.1080/10942912.2010.551310, International Journal of Food Properties, 16(2), 369-381 (2013)

Rojano, B., J. Saez y otros cinco autores, Experimental and theoretical determination of the antioxidant properties of isoespintanol (2-isopropyl-3,6-dimethoxy-5-methylphenol), doi: 10.1016/j.molstruc.2007.07.010, Journal of Molecular Structure, 877, 1-6 (2008)

Santos, S., J. Villaverde y otros cuatro autores, Phenolic composition and antioxidant activity of Eucalyptus grandis: E. urograndis (E. grandis $\times$ E. urophylla) and E. maidenii bark extracts, doi: 10.1016/j.indcrop.2012.02.003, Industrial Crops and Products, 39, 120-127 (2012)

Singh, H., S. Kaur y otros cinco autores, Assessment of in vitro antioxidant activity of essential oil of Eucalyptus citriodora (lemon-scented Eucalypt; Myrtaceae) and its major constituents, doi: 10.1016/j.Iwt.2012.03.019, LWT - Food Science and Technology, 48(2), 237-241 (2012)

Singleton, V. y J. Rossi, Colorimetry of Total Phenolics with Phosphomolybdic-Phosphotungstic Acid Reagents, American Journal of Enology and Viticulture, 16, 144-158 (1965)

Vázquez, G., J. Santos y otros tres autores, Extraction of antioxidants from Eucalyptus (Eucalyptus globulus) bark, doi: 10.1007/s00226-011-0418-y, Wood Science and Technology, 46, 443-457 (2012)

Vuong, Q., H. Sathira y otros cinco autores, Physicochemical, antioxidant and anti-cancer activity of a Eucalyptus robusta (Sm.) leaf aqueous extract, doi: 10.1016/j.indcrop.2014.10.061, Industrial Crops and Products, 64, 167-174 (2015)

Yáñez, X. y O. Mogollón, Composición química y actividad antibacteriana del aceite esencial de las especies Eucalyptus globulus y E. camaldulensis de tres zonas de Pamplona (Colombia), Bistua: Revista de la Facultad de Ciencias Básicas, 10(1), 52-61 (2012)

Zapata, C., L. Duque y otros tres autores, Radical Scavenging Capacity of 2,4-Dihydroxy-9-phenyl 1H phenalen-1-one: A Functional Group Exclusion Approach, doi: 10.1021/ol400384z, Organic Letters 15, 3542-3545 (2013)

Zapata, K., B. Rojano y F. Cortés, Effect of Relative Humidity on the Antioxidant Activity of Spray-Dried Banana Passion Fruit (Passiflora Mollisima Baley)-Coated Pulp: Measurement of the Thermodynamic Properties of Sorption, doi: 10.1080/00986445.2013.840829, Chemical Engineering Communications, 202, 269-278 (2015)

Zambón, S., E. Chamorro y S. Casuscelli, Estudio de la pureza óptica de citronelal presente en los aceites esenciales obtenidos de citronela y de eucalipto citriodora, doi: 10.4067/S0718-07642015000400005, Información Tecnológica, 26(4), 29-36 (2015) 